\title{
Mixing dynamics of dimension-five interactions (scalar/pseudoscalar-photon) in magnetized medium
}

\author{
Ankur Chaubey, Manoj K. Jaiswal, Avijit K. Ganguly
}

\begin{abstract}
In many extentions of standard model, dimension-5 scalar di-photon $\left(g_{\gamma \gamma \phi} \phi\right.$ $\left.F^{\mu \nu} F_{\mu \nu}\right)$ or pseudoscalar di-photon $\left(g_{\gamma \gamma a} a \tilde{F}^{\mu \nu} F_{\mu \nu},\right)$ interaction materializes due to scale symmetry breaking or $U_{A}(1)$ symmetry breaking. In a magnetized vacuum (i.e., in an external background field $\bar{F}_{\mu \nu}$ ) the transverse degrees of freedom of the photons- for such systems- can be described in terms of the form factors constructed out of the background field strength tensor $\left(\bar{F}_{\mu \nu}\right)$ and the same for dynamical photon $\left(f^{\mu \nu}\right)$; they happen to be $\bar{F}_{\mu \nu} f^{\mu \nu}$ and $\tilde{\bar{F}}_{\mu \nu} f^{\mu \nu}$. These form factors transform differently under CP transformation. While $\bar{F}_{\mu \nu} f^{\mu \nu}$ (describing polarization orthogonal to B $\left.\left(\mid \gamma_{\|}>\right)\right)$is CP even, the other one, $\tilde{\bar{F}}_{\mu \nu} f^{\mu \nu}$ (describing polarization along B $\left(\mid \gamma_{\perp}>\right)$, is $\mathrm{CP}$ odd. In the interaction Lagrangian, if the scalar is interchanged with the pseudoscalar, the role of the two form factors just gets interchanged. Thus for nearly degenerate strengths of the coupling constants $\left(g_{\gamma \gamma \phi}\right.$ and $\left.g_{\gamma \gamma_{a}}\right)$ and masses $\left(m_{\phi}\right.$ and $m_{a}$ ) of the respective candidates, proper identification of one from the other may become very difficult in laboratory or astrophysics based experiments. The basic motivation of this investigation is to reduce this uncertainty through incorporation of parity violating (originating through magnetized medium effects ) part of the photon self-energy in the effective Lagrangian. This step, in turn affects the (Pseudo) Scalar Photon mixing dynamics drastically and brings out a significant change in the spectrum of the electromagnetic beam undergoing such interaction.
\end{abstract}

Ankur Chaubey

Institute of Science, Department of Physics, Banaras Hindu University, Varanasi-India.

e-mail: ankur.chaubey@bhu.ac . in

Manoj K. Jaiswal

Department of Physics, University of Allahabad, Prayagraj 211002-India.

e-mail: mkjaiswalbhu@gmail.com

Corresponding author: Avijit K. Ganguly

Department of Physics(MMV), Banaras Hindu University, Varanasi-India.

e-mail: Avijitkganguly@gmail.com 


\section{Introduction}

Pseudoscalar particles like axions a(x) are common to be associated with the breaking of chiral symmetries in many theories of unification (in physics beyond the standard model) [1, 2, 3, 4, 5, 6, 7, 8, 9] through quantum effects; and so is also the case with the Goldstone bosons of a spontaneously broken scale symmetry (dilaton) $\phi(x)[5,6]$. They both have remained possible candidates of Dark matter for some times now. The interaction dynamics of these exotic particles i.e. scalars $(\phi(x))$ or pseudoscalars $(a(x))$ with photon $(\gamma)$ is governed by Dim-5 operators $g_{\gamma \gamma \phi} \phi F^{\mu \nu} F_{\mu \nu}$ or $g_{\gamma \gamma a} a \tilde{F}^{\mu \nu} F_{\mu \nu}$.

The associated form factors $\bar{F}_{\mu \nu} f^{\mu \nu}$ and $\tilde{\bar{F}}_{\mu \nu} f^{\mu \nu}$, for the transverse degrees of freedom of the photons - in external background field $\bar{F}_{\mu \nu}$-for such systems have different $\mathrm{CP}$ transformation properties. As a result, in the equation of motion, the $\mathrm{CP}$ even form factor $\bar{F}_{\mu \nu} f^{\mu \nu}$ couples only to the CP even scalar field $\phi(x)$ while the other CP odd one, $\tilde{\bar{F}}_{\mu \nu} f^{\mu \nu}$, propagates freely. In other words, out of the three available degrees of freedom, the mixing is between only two degrees of freedom- having identical CP properties [10]. And most importantly: the mixing matrix is $2 \times 2$. On the other hand for magnetized pseudo-scalar photon system reverse happens, i.e. the roles of the form factors $\bar{F}_{\mu \nu} f^{\mu \nu}$ and $\tilde{\bar{F}}_{\mu \nu} f^{\mu \nu}$ get interchanged.

Further more, the presence of the external field, compromises the Lorentz symmetry for both the systems; identically. For an external magnetic field in the $z$ direction $\left(B_{z}\right)$, except for rotational and boost symmetry around and along $B_{z}$, all other spacetime symmetries get compromised. This manifests itself by turning the vacuum into an optically active and dichroic medium, for the photons [10, 11, 12] passing through such region. Utilizing this, standard polarimetric observables like polarization or ellipticity angle can be measured and used to determine the magnitude of the coupling constant and mass $g_{\gamma \gamma \phi}$ and $m_{\phi}$ for $\phi-\gamma \gamma$ system or $g_{\gamma \gamma a}$ and $m_{a}$ for $a-\gamma \gamma$ system. This process of determination is however subject to cross-correlated verification from other experiments for example [13].

However, the $2 \times 2$ nature of the mixing matrix for both $\phi(x) \gamma \gamma$ and $a(x) \gamma \gamma$ system poses a problem, when the magnitude of the masses $m_{a}$ and $m_{\phi}$ as well as the coupling constants $\left(g_{\gamma \gamma a}\right)$ and $\left(g_{\gamma \gamma \phi}\right)$ are close to each other. In such a scenario, the identification of one from the other is difficult using the polarimetric techniques. The reason being, as one moves from $\phi(x) \gamma \gamma$ to $a(x) \gamma \gamma$ system, the role of the two polarization form-factors gets interchanged with each other. As a result the absolute magnitude of the ellipticity and polarization angle remain same. And the degree of polarization also remain insensitive to the underlying theory.

The main motivation of this study is to explore other physical corrections, such that the incorporation of them would eventually break the degeneracy in the $2 \times 2$ mixing pattern undergone by both $\phi(x)-\gamma \gamma$ and $a(x)-\gamma \gamma$ system in a magnetized vacuum. 
It so happens that, as one incorporates the parity-violating part of photon-selfenergy-tensor (PSET), that appears once the effect of magnetized medium is incorporated in the evaluation of PSET, in the effective Lagrangian of the system, the apparent degeneracy in mixing gets lifted. This happens due to the discrete symmetries enjoyed by the respective form factors of the photon as well as the scalar or pseudoscalar field. With the incorporation of such effect, the mixing matrix for $\phi F_{\mu \nu} F^{\mu \nu}$, type of interactions, turns out to be $3 \times 3$ and for $a F_{\mu \nu} F^{\mu \nu}$ interaction the mixing matrix is $4 \times 4$. That is there is mixing of all four degrees of freedom -three degrees of freedom of the in-medium photon and one degree of freedom of the pseudoscalar, for $a(x)-\gamma \gamma$ system.

\section{Mixing dynamics of Scalars and Pseudosclars in magnetised plasma}

The action for scalar photon system, as the quantum corrections due to ambient medium and an external magnetic field $\mathbf{e B}$ are taken into account [14], turns out to be

$$
\begin{aligned}
S= & \int d^{4} k\left[\frac{1}{2} A^{v}(-k)\left(-k^{2} \tilde{g}_{\mu \nu}+\Pi_{\mu \nu}(k)+\Pi_{\mu \nu}^{p}(k)\right) A^{\mu}(k)\right. \\
& \left.+i g_{\phi \gamma \gamma} \phi(-k) \bar{F}_{\mu \nu} k^{\mu} A^{\nu}(k)+\frac{1}{2} \phi(-k)\left[k^{2}-m^{2}\right] \phi(k)\right] .
\end{aligned}
$$

Here $\Pi_{\mu \nu}(k)$ is the in medium polarization tensor and $\Pi_{\mu \nu}^{p}(k)$ the parity violating part of the same evaluated in a magnetized medium. One can get the same for pseudoscalar/scalar-photon system from eqn. (1), by replacing $\phi( \pm k)$ by $a( \pm k)$ and $\bar{F}_{\mu \nu}$ by $\tilde{\bar{F}}_{\mu \nu}$. Derivation of the equations of motions follows next.

\subsection{Mixing matrix of Scalar photon interaction}

The equations of motion, of scalar photon system follow from eqn. (1). Written in matrix the same is:

$$
\left[k^{2} \mathbf{I}-\left(\begin{array}{ccc}
\omega_{p}^{2} & i \frac{\omega_{p}^{2} e B_{\|}}{\left(\omega m_{e}\right)} & -i g_{\phi \gamma \gamma} B_{\perp} \omega \\
-i \frac{\omega_{p}^{2} e B_{\|}}{\left(\omega m_{e}\right)} & \omega_{p}^{2} & 0 \\
i g_{\phi \gamma \gamma} B_{\perp} \omega & 0 & m_{\phi}^{2}
\end{array}\right)\right]\left[\begin{array}{c}
A_{\|}(k) \\
A_{\perp}(k) \\
\phi(k)
\end{array}\right]=0 .
$$

The longitudinal degree of freedom, doesn't couple to anything, it propagates freely. The same can be explained with the help of the discrete symmetries enjoyed by the form factor associated with the longitudinal degree of freedom of the photon. Hence the mixing is between $A_{\|}(k), A_{\perp}(k)$ and $\phi$ only. Where $A_{\|}(k), A_{\perp}(k)$ are the form factors associated with the degrees of freedom of photon those are- parallel and 
perpendicular to the direction of magnetic field. We had obtained the solutions of eqn.(2) by diagonalizing the mixing matrix.

\subsection{Mixing matrix of axion photon interaction}

As before the equations of motion for axion photon system, can be expressed in matrix notation as,

$$
\left[\left(\omega^{2}+\partial_{z}^{2}\right) \mathbf{I}-\mathbf{M}^{\prime}\right]\left(\begin{array}{c}
A_{\|}(k) \\
A_{\perp}(k) \\
A_{L}(k) \\
a(k)
\end{array}\right)=0
$$

where $\mathbf{I}$ is an identity matrix and matrix $\mathbf{M}^{\prime}$ is the $4 \times 4$ mixing matrix. The same, in terms of its elements is given by,

$$
\mathbf{M}^{\prime}=\left(\begin{array}{cccc}
\Pi_{T} & -\Pi_{p} N_{1} N_{2} P_{\mu \nu} b^{(1) \mu} I^{\nu} & 0 & 0 \\
\Pi_{p} N_{1} N_{2} P_{\mu \nu} b^{(1) \mu} I^{\nu} & \Pi_{T} & 0 & -i g_{a \gamma \gamma} N_{2} b_{\mu}^{(2)} I^{\mu} \\
0 & 0 & \Pi_{L} & -i g_{a \gamma \gamma} N_{L} b_{\mu}^{(2)} \tilde{u}^{\mu} \\
0 & i g_{a \gamma \gamma} N_{2} b_{\mu}^{(2)} I^{\mu} & i g_{a \gamma \gamma} N_{L} b_{\mu}^{(2)} \tilde{u}^{\mu} & m_{a}^{2}
\end{array}\right) .
$$

We note that, projection operator $P_{\mu \nu}$, appearing in the $M_{12}^{\prime}$ and $M_{21}^{\prime}$ elements of the mixing matrix $M^{\prime}$ is a complex one, that makes the matrix, $M^{\prime}$, a hermitian matrix, that is expected even otherwise on general grounds.

It is also important to note that for pseudoscalar-photon interaction, because of discrete symmetry considerations (PT symmetry to be specific )the form factor associated with longitudinal degree of freedom remains coupled with pseudoscalar field. Hence the mixing matrix becomes $4 \times 4$. Therefore the mixing dynamics for these two systems with incorporation of parity violating medium effect, turns out to be completely different. Due to this, the identification of one from the other using polarimetric observables may become lot easier.

\section{Optical observables}

Properties of polarized light waves can be described in terms of the Stokes parameters evaluated from the coherency matrix. The same is constructed from the solutions of the field equations; and is given by:

$$
\mathbf{D}^{\prime}(z)=\left(\begin{array}{c}
<\mathbf{A}_{\|}(\omega, \mathbf{z}) \mathbf{A}_{\|}^{*}(\omega, \mathbf{z})><\mathbf{A}_{\|}(\omega, \mathbf{z}) \mathbf{A}_{\perp}^{*}(\omega, \mathbf{z})> \\
<\mathbf{A}_{\perp}(\omega, \mathbf{z}) \mathbf{A}_{\|}^{*}(\omega, \mathbf{z})><\mathbf{A}_{\perp}(\omega, \mathbf{z}) \mathbf{A}_{\perp}^{*}(\omega, \mathbf{z})>
\end{array}\right) .
$$


In eqn. (5) above, <> represent the ensemble averages. The Stokes parameters are obtained from the elements of the coherency matrix by the following identifications; $\mathbf{I}=D_{11}^{\prime}(z)+D_{22}^{\prime}(z), \mathbf{Q}=D_{11}^{\prime}(z)-D_{22}^{\prime}(z), \mathbf{U}=2 \operatorname{Re} D_{12}^{\prime}(z)$, and $\mathbf{V}=2 \operatorname{Im} D_{12}^{\prime}(z)$.

The estimates of the other optical parameters, i.e., ellipticity angle, polarization angle, degree of linear polarization, degree of total polarization, follows from the expressions of $\mathbf{I}, \mathbf{U}, \mathbf{Q}$ and $\mathbf{V}$. The expressions for the polarization angle and ellipticity angle, associated with an electromagnetic wave are provided below.

\subsection{Polarization Angle \& Ellipticity Angle}

Polarization angle (represented by $\Psi$ ) is the angle between major and minor axis of ellipse, defined in terms of stokes parameters $\mathbf{U}$ and $\mathbf{Q}$, is given by,

$$
\tan (2 \Psi)=\frac{\mathbf{U}(\omega, z)}{\mathbf{Q}(\omega, z)}
$$

The ellipticity angle (denoted by $\chi$ ) is defined in terms of the same parameters as,

$$
\tan (2 \chi)=\frac{\mathbf{V}(\omega, z)}{\sqrt{\mathbf{Q}^{2}(\omega, z)+\mathbf{U}^{2}(\omega, z)}} .
$$

\section{Results and Conclusions}

Unlike polarization angle, the ellipticity angle remains invariant under rotation of the axes. So we have compared the magnitude of the ellipticity angle produced through axion-photon as well as scalar-photon interaction, in the vicinity of a strongly magnetized compact astrophysical source. The parameters, that we have considered for the system are as follows: plasma frequency $\omega_{p}=1.6 \times 10^{-10} \mathrm{GeV}$, coupling constants $g_{\gamma \gamma a}=g_{\gamma \gamma \phi}=10^{-11} \mathrm{GeV}^{-1}$ and mass $m_{a}$ and $m_{\phi}$ both close to zero. The magnetic field is taken to be $B=10^{12}$ Gauss and the path length considered here is $2.5 \mathrm{Km}$.. The numerical estimates of the ellipticity angle for the two systems are plotted in, Fig.[1].

As can be seen in the plot that - for the values of the parameters chosen here - the numerical magnitudes of the angle for the $a \gamma$ and $\phi \gamma$ system, are extremely close to each other. However there is some departure, that can be seen in the inset of Fig.[1]. In the energy range of, $1 \times 10^{-5} \mathrm{GeV}$ to $1.5 \times 10^{-5} \mathrm{GeV}$, there is some visible difference in the ellipticity angle between axion photon and scalar photon systems. For energies close $1 \times 10^{-5} \mathrm{GeV}$ the difference is around $3 \times 10^{-7} \mathrm{rad}$. Though this is little less for current sensitivity available for the detectors, however we hope that, future detectors would have similar sensitivity to resolve this difference and shed light on the values of the parameters like $g_{\gamma \gamma \phi}$ or $g_{\gamma \gamma a}$ and $m_{\phi}$ or $m_{a}$. Studies along 
this direction are currently under progress and would be communicated else where shortly.

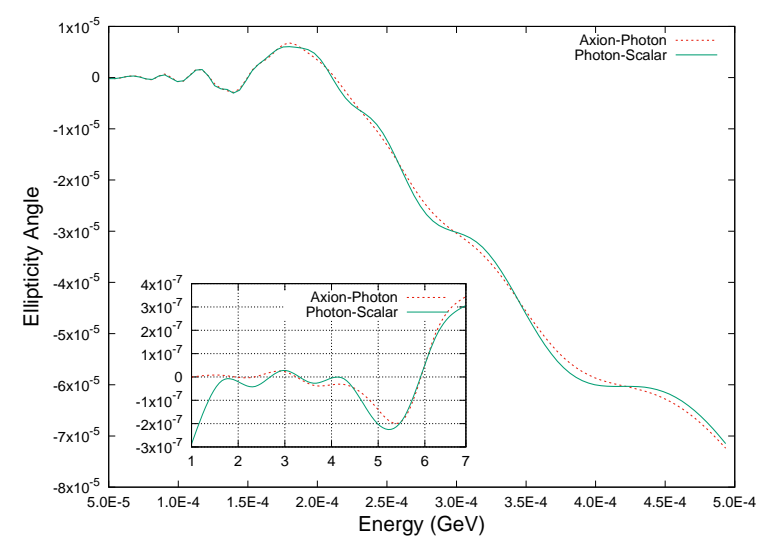

Fig. 1 Plot for ellipcity angle vs energy in case of coupled photon-axion system. The abscissa of the plot, in the inset is, in units of $10^{-5} \mathrm{GeV}$.

\section{References}

1. R.D. Peccei and H.R. Quinn, Phys. Rev. Lett. 38, 1440 (1977); Phys. Rev. D 16, 1791 (1977). R.D. Peccei, Report No. hep-ph/9606475 for a more extensive review of the strong CP problem, see in CP Violation, edited by C. Jarlskog (World Scientific, Singapore, 1989). R. D. Peccei and H. R. Quinn, Phys. Rev. Lett. 38, 1440 (1977).

2. S. Weinberg, Phys. Rev. Lett. 40, 223 (1978).

3. F. Wilczek,Phys. Rev. Lett. 40, 279 (1978).

4. Y. Chikashige, R. Mohapatra, and R.D. Peccei, Phys. Rev. Lett. 45, 19261980 ; Phys. Lett. 98B, 2651981 ; G. Gelmini and M. Roncadelli, ibid. 99B, 4111981.

5. Y. M. Cho and J. H. Kim Phys. Rev. D 79023504 (2009).

6. T. Damour and J. F. Donoghue, Phys. Rev. D 79023504 (2009).

7. P. Svrcek and E. Witten, JHEP 06061 (2006).

8. Ashok Das,Jnanadeva Maharana, Phys Lett. B 699, 264 (2011). https://doi.org/10.1016/

9. D.J.E.Marsh, Phys. Rept. 643, 1 (2016)

10. G. Raffelt and L. Stodolsky, Phys. Rev. D 371237 (1988).

11. L. Maiani, R. Petronzio and E. Zavattini, Phys. Lett. B 175, 359 (1986).

12. A. K. Ganguly and M. K. Jaiswal Phys. Rev. D 90026004 (2014).

13. M. Lawson, A. J. Millar, E. Vitagliano, F. Wilczek, Tunable Axion Plasma haloscopes (arXiv:1904, 11872[hep-ph]).

14. A. K. Ganguly, P. Jain, and S. Mandal, Phys. Rev. D 79, 115014 (2009) 